\title{
Geostatistical modeling of topography using auxiliary maps
}

\author{
Tomislav Hengl ${ }^{\mathrm{a}, *}$, Branislav Bajat ${ }^{\mathrm{b}}$, Dragan Blagojević $^{\mathrm{b}}$, Hannes I. Reuter $^{\mathrm{c}}$ \\ ${ }^{a}$ Institute for Biodiversity and Ecosystem Dynamics, University of Amsterdam, Nieuwe Achtergracht 166,1018 WV Amsterdam, The Netherlands \\ ${ }^{\mathrm{b}}$ Faculty of Civil Engineering, Bulevar kralja Aleksandra 73, 11000 Belgrade, Serbia \\ ${ }^{\mathrm{c}}$ European Commission, Directorate General JRC, Institute for Environment and Sustainability, TP 280, Via E. Fermi 1, I-21020 Ispra (VA), Italy
}

\section{A R T I C L E I N F O}

\section{Article history:}

Received 29 May 2007

Received in revised form

22 December 2007

Accepted 3 January 2008

\section{Keywords:}

DEM

$\mathrm{R}$

Variogram

Regression-kriging

Error

Simulations

Elevation

\begin{abstract}
A B S T R A C T
This paper recommends computational procedures for employing auxiliary maps, such as maps of drainage patterns, land cover and remote-sensing-based indices, directly in the geostatistical modeling of topography. The methodology is based on the regressionkriging technique, as implemented in the $\mathrm{R}$ package gstat. The computational procedures are illustrated using a case study in the south-west part of Serbia. Two point data sets were used for geostatistical modeling: (1) 2051 elevation points were used to generate DEMs and (2) an independent error assessment data set (1020 points) was used to assess errors in the topo-DEM and the SRTM-DEM. Four auxiliary maps were used to improve generation of DEMs from point data: (1) distance to streams, (2) terrain complexity measured by standard deviation filter, (3) analytical hillshading map and (4) NDVI map derived from the Landsat image. The auxiliary predictors were significantly correlated with elevations $\left(\operatorname{adj} . R^{2}=0.20\right)$ and DEM errors $\left(\operatorname{adj} . R^{2}=0.27\right)$. By including auxiliary maps in the geostatistical modeling of topography, realizations of DEMs can be generated that represent geomorphology of a terrain more accurately. In addition, downscaling of a coarse 3 arcsec SRTM DEM using auxiliary maps and regression-kriging is demonstrated using the same case study. A methodological advantage of regression-kriging, compared to splines, is the possibility to automate the data processing and incorporate multiple auxiliary predictors. The remaining open issues are computational efficiency, application of local regression-kriging algorithms and preparation of suitable auxiliary data layers for such analyses.
\end{abstract}

(c) 2008 Elsevier Ltd. All rights reserved.

\section{Introduction}

A digital elevation model (DEM) is a digital representation of the land surface-the major input to quantitative analysis of topography, also known as digital terrain analysis or geomorphometry (Evans, 1972; Pike, 1995; Wilson and Gallant, 2000; Hengl and Reuter, 2008). Typically, a DEM is a raster map (an image or an elevation array) that, like many other spatial features, can be efficiently modeled using geostatistics. The geostatistical concepts were introduced in geomorphometry by Fisher $(1992,1998)$ and Wood and Fisher (1993), then further elaborated by Kyriakidis et al. (1999), Holmes et al. (2000) and Oksanen (2006b). The methodological developments and future trends of geostatistical modeling of topography can be followed in the Ph.D. thesis of Oksanen (2006a).

An important focus of using geostatistics to model topography is assessment of the errors in DEMs and analysis of effects that the DEM errors have on the results of spatial modeling. This is the principle of error propagation that commonly

\footnotetext{
* Corrresponding author. Tel.:+31205257458; fax:+31 205257431.

E-mail addresses: hengl@science.uva.nl (T. Hengl), bajat@grf.bg.ac.yu (B. Bajat), bdragan@grf.bg.ac.yu (D. Blagojević), hannes.reuter@jrc.it (H.I. Reuter).
} 
works as follows: simulations are generated from point-measured heights to produce multiple equiprobable realizations of a DEM of an area; a spatial model is applied $m$ times and output maps then analyzed for mean values and standard deviations per pixel; the results of analysis can be used to quantify DEM accuracy and observe impacts of uncertain information in various parts of the study area (Hunter and Goodchild, 1997; Heuvelink, 1998; Oksanen and Sarjakoski, 2005; Raaflaub and Collins, 2006). An animated illustration of an error propagation study for derivation of slope, curvatures, solar insolation and northness maps is available at www.geomorphometry.org (under DEM simulations).

So far, DEMs have been generated by using solely point-sampled elevations. For example, ordinary kriging is used to generate DEMs (Mitas and Mitasova, 1999; Lloyd and Atkinson, 2002); conditional geostatistical simulations are used to generate equiprobable realizations of DEMs (Fisher, 1998; Kyriakidis et al., 1999). In most studies, no auxiliary (also known as additional or secondary) information on variation of relief is employed directly in the geostatistical modeling. Compared to the approach of Hutchinson (1989) or Hutchinson (1996) where auxiliary maps of streams are often used to produce hydrologically correct DEMs, the geostatistical approach to modeling of topography has often been limited to analysis of point-sampled elevations.

Our interest in this paper is to develop and test a more advanced methodology to model topography using geostatistics by including the auxiliary maps directly into the geostatistical analysis. By auxiliary maps, we consider all GIS layers that can explain spatial distribution of measured elevations and associated errors. Our assumption is that, by including such information, we will be able to produce more accurate realizations of DEMs and, consequently, advance the use of geostatistics in geomorphometry.

\section{Theory}

A DEM can be defined as an elevation array with a (large) number of grid nodes over the domain of interest:

$$
\mathbf{Z}=\left\{Z\left(\mathbf{s}_{j}\right), j=1, \ldots, N\right\} ; \quad \mathbf{s}_{j} \in \mathbb{A}
$$

where $\mathbf{Z}$ is the elevation array, $Z\left(\mathbf{s}_{j}\right)$ is the elevation at the grid node $\mathbf{s}_{j}$, $\mathbb{A}$ is the area of interest and $N$ is the total number of grid nodes. DEMs are today increasingly produced using automated (mobile GPS) field sampling of elevations or airborne scanning devices (radar or LiDAR-based systems). In the case elevations are sampled at sparsely located points, a DEM can be generated using geostatistical techniques such as ordinary kriging (Wood and Fisher, 1993; Mitas and Mitasova, 1999). The elevation at some grid node $\left(\mathbf{s}_{0}\right)$ of the output DEM can be interpolated using:

$$
\hat{Z}\left(\mathbf{s}_{0}\right)=\mathbf{c}_{\mathbf{0}}^{\mathbf{T}} \times \mathbf{C}^{-1} \times \mathbf{z}
$$

where $\mathbf{c}_{\mathbf{0}}^{\mathbf{T}}$ is the covariance vector at the prediction location, $\mathbf{C}$ is the covariance matrix at sampled locations $\mathbf{s}_{i}$ and $\mathbf{z}$ is an array of sampled points $\left(z\left(\mathbf{s}_{i}\right) ; i=1, \ldots, n\right)$. The covariances are determined by fitting a variogram model $\gamma(\mathbf{h})$, where $\mathbf{h}$ is the distance between the point pairs of sampled elevations.

The same technique (kriging) can be used to produce simulated DEMs. An equiprobable realization of a DEM can be generated by using the sampled elevations and their variogram model:

$$
Z^{(\mathrm{SIM})}\left(\mathbf{s}_{0}\right)=E\left\{Z \mid Z\left(\mathbf{s}_{j}\right), \gamma(\mathbf{h})\right\}
$$

where $Z^{(\mathrm{SIM})}$ is the simulated value at the prediction location. The most common technique in geostatistics that can be used to generate equiprobable realizations is the Sequential Gaussian Simulation (Goovaerts, 1997, pp. 380-392). It starts by defining a random path for visiting each node of the grid once. At first node, kriging is used to determine the locationspecific mean and variance of the conditional cumulative distribution function. A simulated value can then be drawn by using the inverse normal cumulative distribution function, e.g. (Box and Muller, 1958):

$$
z^{\operatorname{SIM}}\left(\mathbf{s}_{0}\right)=\hat{z}\left(\mathbf{s}_{0}\right)+\hat{\sigma}\left(\mathbf{s}_{0}\right) \cdot \sqrt{-2 \cdot \ln (1-A)} \cdot \cos (2 \cdot \pi \cdot B)
$$

where $z^{\mathrm{SIM}}$ is the simulated elevation, $A$ and $B$ are the independent random numbers within the $0-0.99 \ldots$ range, $\hat{z}$ is the predicted (mean) value at $\mathbf{s}_{0}$ location and $\hat{\sigma}$ is the prediction error at that location. The simulated value is then added to the original data set and the procedure is repeated until all nodes have been visited. Direct simulation of DEMs using the sampled elevations is discussed in detail by Kyriakidis et al. (1999).

If additional, auxiliary maps (drainage network, water bodies, physiographic break-lines) are available, a DEM can be generated from the point-measured elevations using the regression-kriging model (Christensen, 2001, p. 277):

$$
\hat{Z}\left(\mathbf{s}_{0}\right)=\mathbf{q}_{\mathbf{0}} \times \hat{\boldsymbol{\beta}}^{\mathbf{T}}+\mathbf{C}_{\mathbf{0}}^{\mathbf{T}} \times \mathbf{C}^{-1} \times\left(\mathbf{z}-\mathbf{q} \times \hat{\boldsymbol{\beta}}^{\mathbf{T}}\right)
$$

where $\mathbf{q}_{\mathbf{0}}$ is the vector of predictors at new location, $\hat{\boldsymbol{\beta}}$ is the vector of fitted regression coefficients and $\mathbf{q}$ is the matrix of predictor values at sampled locations.

The biggest advantage of using auxiliary maps is a possibility to more precisely model uncertainty of the sampled elevations and analyze which external factors cause this variability. Whereas, in pure statistical Monte Carlo approach where we work with global, constant parameters (Fig. 1a), in the case of geostatistical modeling, the DEM uncertainty can be modeled with a much higher level of detail (Fig. 1b). 


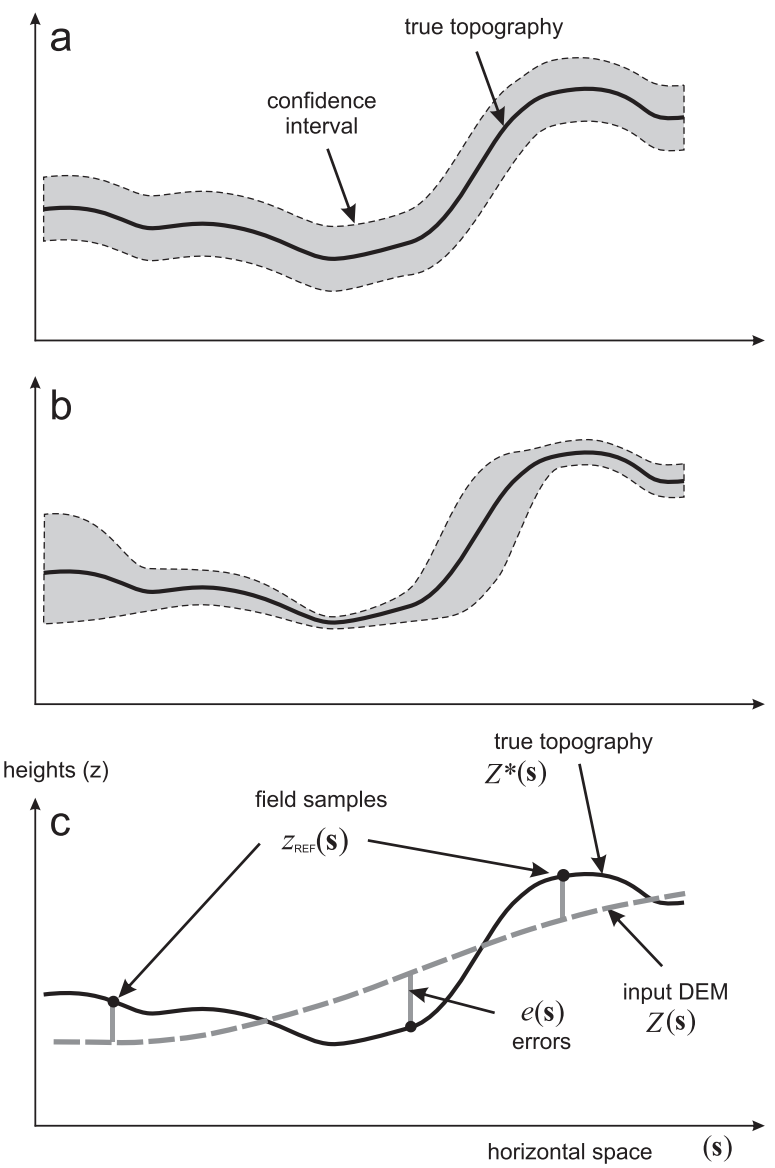

Fig. 1. Conceptual aspects of modeling topography using geostatistics. A cross-section showing true topography and associated uncertainty: (a) constant, global uncertainty model and (b) spatially variable uncertainty; (c) estimation of DEM errors using precise height measurements (usually based on field sampling).

In the case a DEM is obtained from an airborne or satellite-based scanning mission (radar, LiDAR or stereoscopic images), elevations are already available over the whole area of interest. Geostatistics is then used to analyze inherent errors in the DEM images (Grohmann, 2004), filter local errors caused by physical limitations of the instrument (Lloyd and Atkinson, 2002; Evans and Hudak, 2007), and eventually cluster the area according to their statistical properties (Lloyd and Atkinson, 1998).

Geostatistical simulation of complete elevation data is somewhat more complicated than with point data. At the moment, the simulations of DEM images are most commonly obtained by simulating error surfaces derived from additional field-control samples (Fig. 1c). The elevations measured at control points are used to assess the errors. The point map of DEM errors can then be used to generate equiprobable error surfaces, which are then added to the original DEM to produce an equiprobable realization of a DEM (Hunter and Goodchild, 1997; Holmes et al., 2000; Endreny and Wood, 2001; Temme et al., 2008). This technique is based on the following theory. From a statistical perspective, a DEM produced directly by using scanning devices (SRTM, LiDAR) consists of three components:

$$
Z\left(\mathbf{s}_{j}\right)=Z^{*}\left(\mathbf{s}_{j}\right)+\varepsilon^{\prime}\left(\mathbf{s}_{j}\right)+\varepsilon^{\prime \prime}
$$

where $Z^{*}\left(\mathbf{s}_{j}\right)$ is the deterministic component, $\varepsilon^{\prime}\left(\mathbf{s}_{j}\right)$ is the spatially correlated random component and $\varepsilon^{\prime \prime}$ is the pure noise, usually the result of the measurement error. In raster-GIS terms, we can decompose a DEM into two grids: (1) the deterministic DEM and (2) the error surface. If precise point samples of topography (e.g. highly precise GPS measurements) are available, they can be used to estimate the errors (Fig. 1c):

$$
e\left(\mathbf{s}_{i}\right)=z_{\mathrm{REF}}^{*}\left(\mathbf{s}_{i}\right)-Z\left(\mathbf{s}_{i}\right) ; \quad E\{e(\mathbf{s})\}=0
$$

The measured errors at point locations can also be manipulated using geostatistics to generate the error surface:

$$
e^{(\mathrm{SIM})}\left(\mathbf{S}_{0}\right)=E\left\{\varepsilon \mid e\left(\mathbf{s}_{i}\right), \gamma_{e}(\mathbf{h})\right\}
$$


The simulated error surface can then be added to the deterministic DEM to produce an equiprobable realization of a DEM:

$$
Z^{(\mathrm{SIM})}\left(\mathbf{s}_{j}\right)=Z^{*}\left(\mathbf{s}_{j}\right)+e^{(\mathrm{SIM})}\left(\mathbf{s}_{j}\right)
$$

An obvious problem with this approach is that the deterministic DEM $\left(Z^{*}\left(\mathbf{s}_{j}\right)\right)$ is usually not available, so that the input DEM is in fact used to generate simulations, which leads to (see, e.g., Holmes et al., 2000; Temme et al., 2008):

$$
\begin{aligned}
Z^{(\mathrm{SIM})}\left(\mathbf{s}_{j}\right) & =Z\left(\mathbf{s}_{j}\right)+e^{(\mathrm{SIM})}\left(\mathbf{s}_{j}\right) \\
& =Z^{*}\left(\mathbf{s}_{j}\right)+\varepsilon^{\prime}\left(\mathbf{s}_{j}\right)+\varepsilon^{\prime \prime}+e^{(\mathrm{SIM})}\left(\mathbf{s}_{j}\right)
\end{aligned}
$$

This means that the simulated error surface and the inherent error component, at some locations, will double, and at others will annul each other. However, because the inherent error and the simulated error are in fact independent, the mean of the summed errors will be close to zero (unbiased simulation), but the standard deviation of the error component will be on average 40\% larger. Hence a DEM simulated using Eq. (9) will be much noisier than the original DEM. The solution to this problem is to substitute the deterministic DEM component with a smoother DEM, e.g. a DEM derived from contour lines digitized from a finer-scale topo-map. As an alternative, the deterministic DEM component can be prepared by smoothing the original DEM, i.e. filtering it for known noise and systematic errors (see, e.g., Selige et al., 2006). The more robust, more sophisticated solutions are yet to be developed.

The spatially correlated error component will also often correlate with the auxiliary information (Carlisle, 2005; Oksanen, 2006b). For example, in traditional cartography, it is known that the error of measuring elevations is primarily determined by the complexity of terrain (the slope factor), land cover (density of objects) and relative visibility (the shadow effect). Especially, in the cases where the DEMs are produced through photogrammetric methods, information about the terrain shading can be used to estimate the expected error of measuring heights. Similarly, an SRTM DEM will show systematic errors in areas of higher canopy and smaller precision in areas which are hidden or poorly exposed to the scanning device (Hengl and Reuter, 2008). This opens a possibility to also use the regression-kriging model with auxiliary maps to produce a more realistic error surface (Eq. (5)).

\section{Methods and materials}

\subsection{Case study}

We used a case study area "Zlatibor" located in the south-western part of Serbia (centered at $43^{\circ} 43^{\prime} 44.6^{\prime \prime} \mathrm{N}$ and $19^{\circ} 42^{\prime} 37.8^{\prime \prime} \mathrm{E}$ ). The area is mainly hilly plateau, with the exception of the north-east part where the slopes are much steeper (Fig. 2a). Elevations range from $850 \mathrm{~m}$ to a maximum of $1174 \mathrm{~m}$; the total size of the area is $13.5 \mathrm{~km}^{2}$. The data set consists of four input height-data: (a) a set of 2051 height measurements used for generation of DEMs (elevations), (b) a set of 1020 very precise spot heights used for error assessment (control), (c) the original topo-map DEM at $30 \mathrm{~m}$ resolution and (d) $3 \operatorname{arcsec}(\sim 90 \mathrm{~m})$ SRTM DEM.

The original topo-map DEM was produced by digitizing contour layers from two adjacent sheets of the 1:5000 topographic maps with contour interval of $5 \mathrm{~m}$. Two sheets were scanned by ANATech Evolution scanner with 400 DPI resolution, then georeferenced to the Gauss-Krüger coordinate system (7th zone) and converted to a point map using a semi-automated digitalization of contour lines (I/GEOVEC, Intergraph program module). The faults obtained during automated digitalization were removed by 3D editing of contour lines. The final DEM was produced using the ArcGIS 3D analyst: first a TIN was produced, which was then converted to a regular grid of $30 \mathrm{~m}$ resolution. This will be referred to as the topo-DEM in further text. The original points extracted from the topo-map (51,847 points) were sub-sampled (for computational efficiency) to 2051 points.

A set of 1020 photogrammetric control points was provided with the help of the Geodetic governmental authority of Serbia. These were obtained throughout the orthophoto map production of the scale 1:1000 for local municipality. Aerial images were obtained using the RMK 21/23 analog camera with calibrated focal length $f=207.96 \mathrm{~mm}$. The average flying altitude was $1040 \mathrm{~m}$. A stereoscopic model was produced using the WILD A10 analog stereo-restitution instrument and MapSoft 2000 program package. The land-surface points were measured manually from the stereoscopic model with average lag of $15 \mathrm{~m}$. This gave a total number of 46,021 points that were sub-sampled to 1020 points for faster processing. The estimated height accuracy of control points is $\pm 15 \mathrm{~cm}$ which allows us to use it as ground truth for the topo-DEM.

\subsection{Auxiliary maps}

We have considered four auxiliary variables that we found of potential interest for modeling topography: (a) distance to streams (dstreams), (b) terrain complexity (stdev), (c) analytical hillshading (shade) and (d) remote-sensing-based vegetation index (NDVI). Distance to streams was derived by, first digitizing the stream lines from the topo map, and then running a distance operation in ILWIS GIS (www. 52 north.org). The terrain complexity was estimated using a $5 \times 5$ window standard deviation filter. This produces a map very similar to the slope gradient map and can be used to quantify the complexity of terrain. Analytical hillshading was derived using standard sun position settings (sun inclination at $45^{\circ}$, azimuth at $315^{\circ}$ ) for the geographic area. Both terrain complexity and analytical hillshading were derived in SAGA GIS 


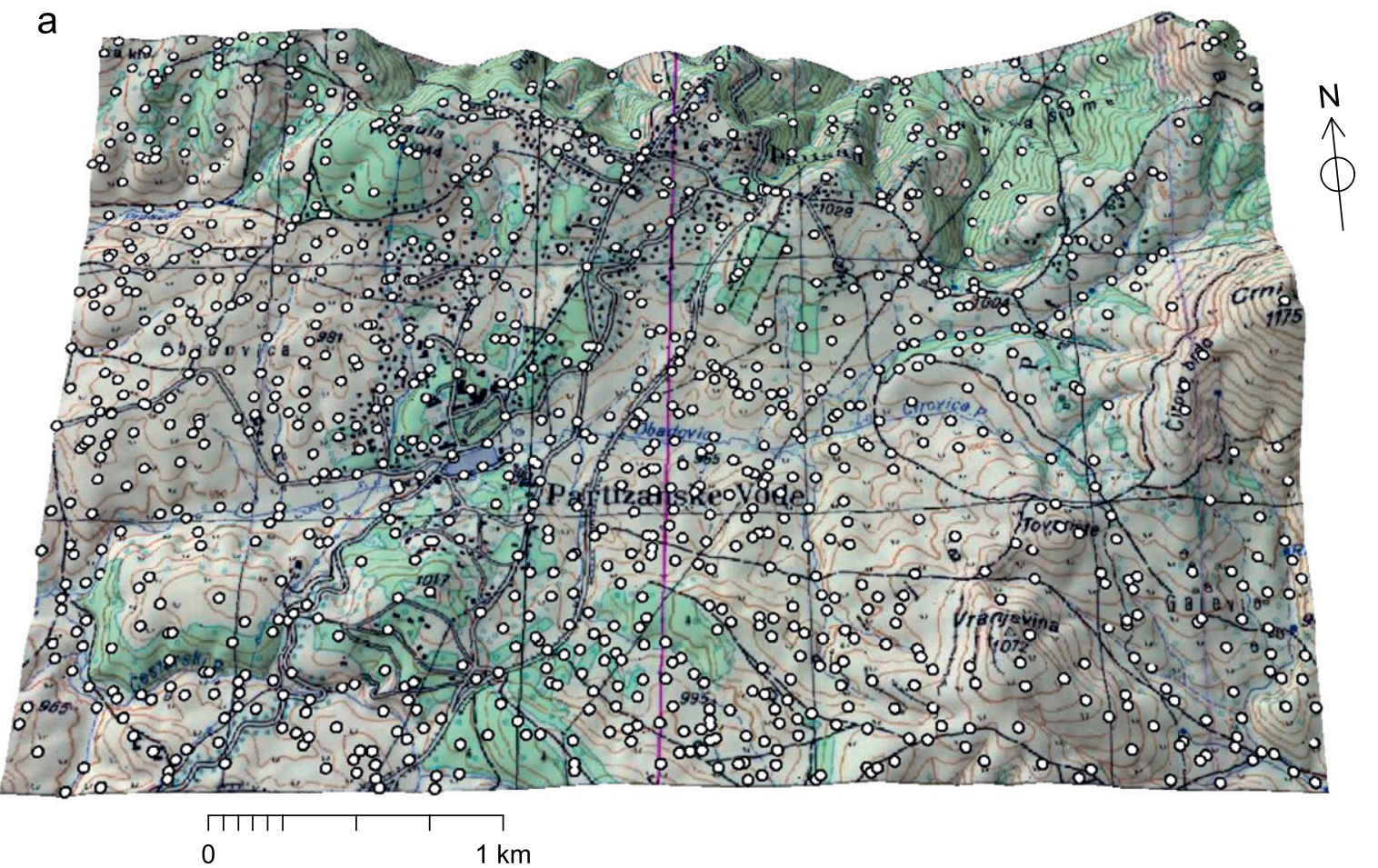

b
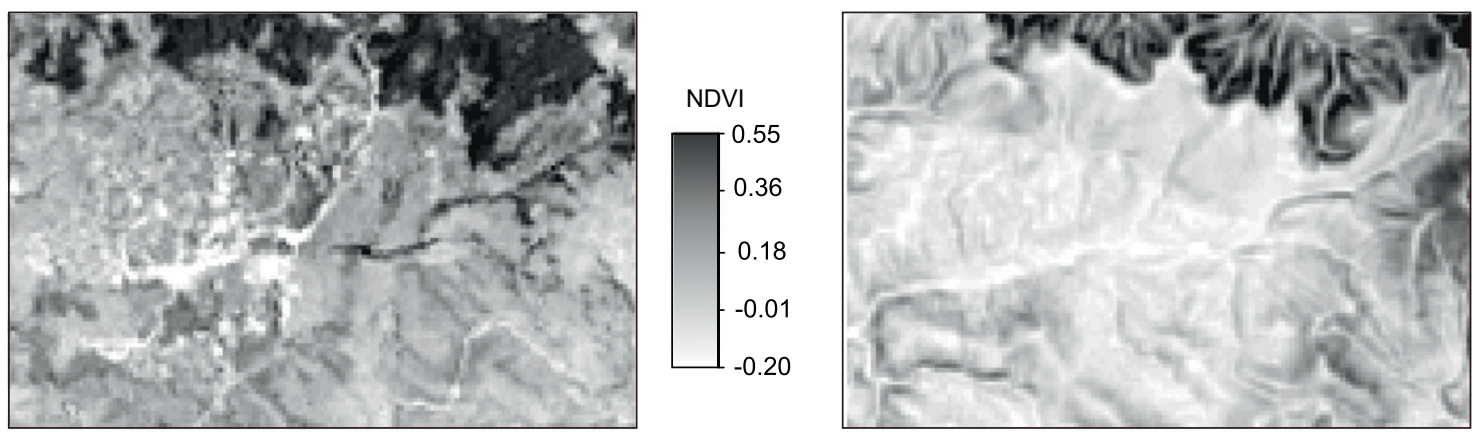

stdev
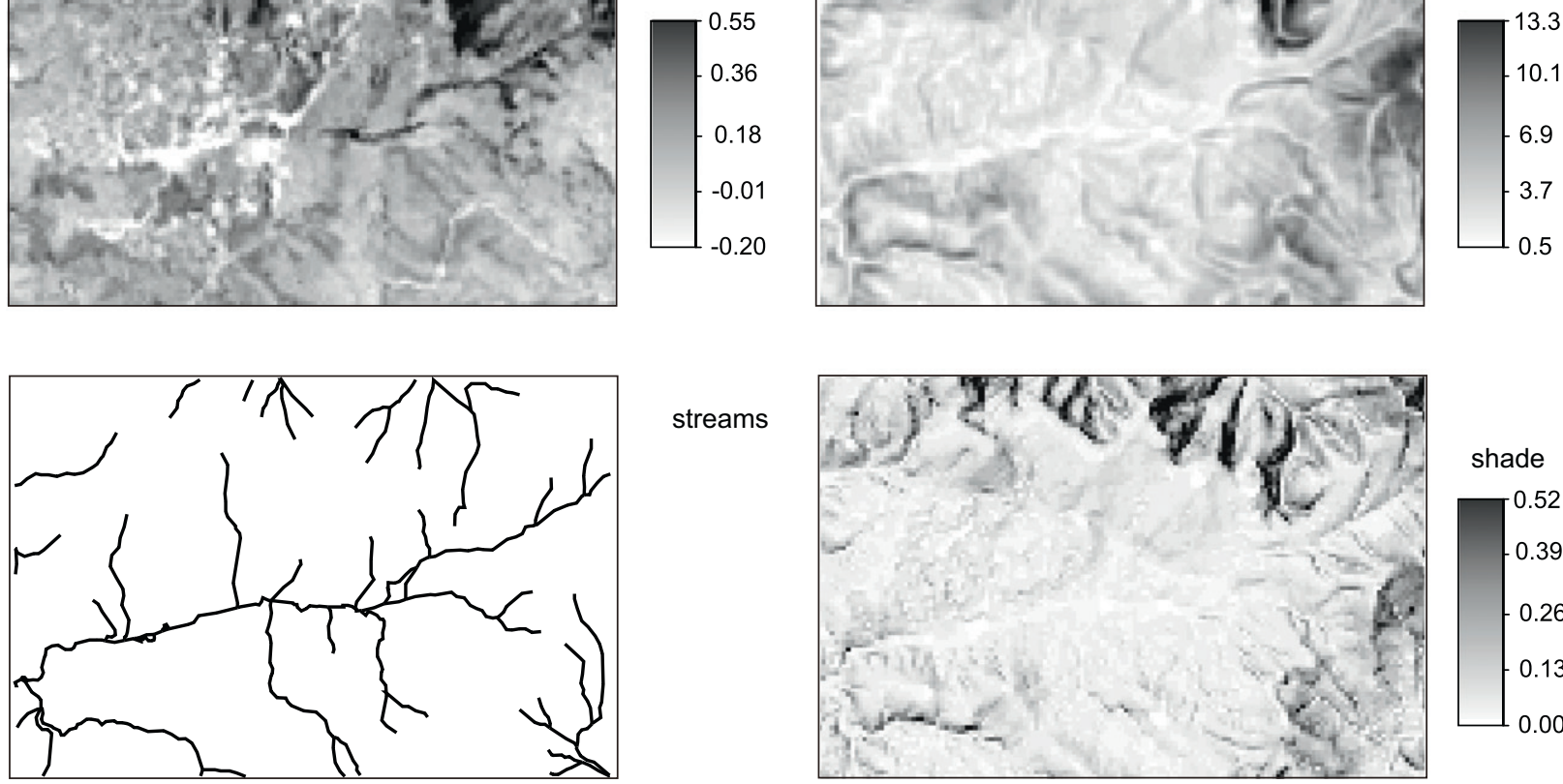

streams

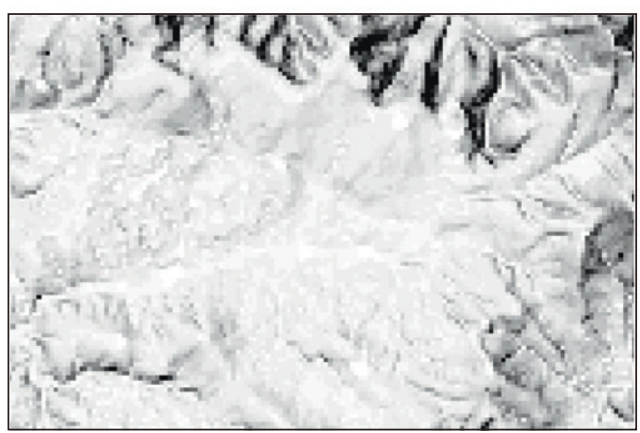

shade

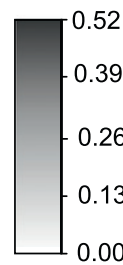

Fig. 2. Study area Zlatibor: (a) perspective view on area (1:25,000 topo-map) and location of 1020 error assessment points, (b) a preview of auxiliary predictors used for geostatistical modeling.

(www. saga-gis.org) using the existing $30 \mathrm{~m}$ DEM. NDVI was derived from the Landsat 7 ETM image, which was obtained from the Global Land Cover Facility of the University of Maryland (ftp.glcf.umiacs.umd.edu). A preview of all auxiliary maps used in this exercises can be seen in Fig. $2 \mathrm{~b}$. 


\subsection{Data processing in $R$}

The geostatistical analysis of topography can be efficiently implemented using the R statistical computing environment and gstat and sp packages, kindly provided by Edzer Pebesma and collaborators (Pebesma, 2004; Pebesma and Bivand, 2005). The GIS layers, grids and point maps, can be imported in R using the rgdal package, or used directly in a GIS through a link to R, e.g. in GRASS GIS (Grohmann, 2004). The sp package is used to define the spatial data and visualize the maps.

The 3D point table $(x, y, z)$ needs to be first imported to $\mathrm{R}$ and converted to a spatial data frame using:

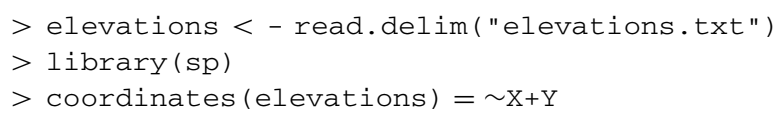

A DEM can now be generated directly from the sampled data using the gstat package:

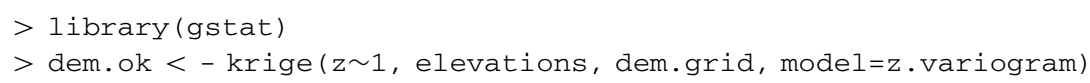

where dem. ok is the output data frame, krige is the generic kriging command ( $z \sim 1$ indicates that ordinary kriging will be used), elevations is the point data set of sampled elevations, dem.grid is the grid definition for new predictions and $z$. variogram is the fitted variogram model for sampled elevations. We can easily switch from estimation to simulations by adding to the command above an additional argument: nsim=1. Multiple simulations can be generated by increasing the number set for this argument.

Raster maps can be imported to $\mathrm{R}$ using the rgdal package:

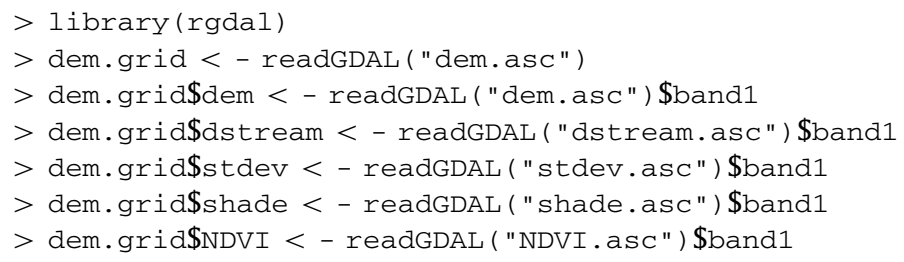

And values of predictors estimated using the overlay function in $\mathrm{R}$, for example:

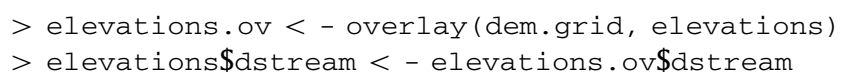

Now, also auxiliary maps can be used to generate DEMs by extending the formula argument:

$>$ dem.sim < - krige(z dstreams, elevations, dem.grid, model=zres.variogram, nsim=1)

This will produce a realization of a DEM by taking into account the distance to streams map (dstreams) and regressionkriging model (Eq. (5)). Note also that we need to know the variogram model for the residuals, which can be estimated automatically using:

> zres.variogram< - fit.variogram(variogram(z〜dstreams, elevations), vgm(1, "Sph", 1000, 1))

where vgm $(1$, "Sph" , 1000, 1) is the initial variogram model set by the user.

Regression-kriging can also be used to simulate the error surfaces. For example, assuming that the errors are controlled by the terrain complexity (stdev), shading effect (shade) and/or canopy height (NDVI), the command to simulate the error surface using an error assessment point data set (control):

error.sim < - krige (enstdev+shade+NDVI, control, dem.grid, model=eres.variogram, nsim=1)

All output maps can be exported to GIS packages (including Google Earth) by using the rgdal and maptools packages. For more information on the use of these tools see Hengl (2007). All scripts and data sets used in this paper can be obtained from the www.geomorphometry.org website. 


\section{Results}

\subsection{DEM generation using auxiliary maps}

We first fitted a variogram model to the sampled elevations data set (elevations) using a spherical variogram model with nugget parameter $C_{0}=0$, a sill parameter of $C_{1}=1561$ and the range parameter of $1235 \mathrm{~m}$ (Fig. 3a). Elevations follow a normal distribution with a mean of $993 \mathrm{~m}$ and standard deviation of $46 \mathrm{~m}$ (Fig. 3b). Both properties make this data set suitable for linear geostatistical modeling without additional transformations.

The two predictors (dstreams and NDVI) explain 20\% of the total variation in the 2051 point measurements. Both predictors are significant at 0.001 probability levels. The variogram model of the elevations, after using the two predictors, has now about 30\% smaller sill (1244) and a similar range (1202 m) (Fig. 3a). However, relationship between elevation and NDVI is not completely clear (Fig. 3c) -it seems that high NDVI values are clearly observed at low elevations, but low NDVI values can be observed at both high and low elevations. Conceptually speaking, there is no reason why the elevations should decrease with higher NDVI. Locally, only inside this area, we know that the deeper natural forests occur at the areas of lower elevation. Hence, NDVI should be used with caution or only to explain variation in the elevations locally.

The results of generating predictions and simulations from sampled elevations without and with using the auxiliary maps can be seen in Fig. 4. If auxiliary maps are used, the output DEM (Fig. 4b) will follow the drainage patterns and the geomorphology will be presented more precisely than when pure ordinary kriging (Fig. 4a) is used. In the case of simulations, it is harder to see the difference between the two DEMs (Fig. 4c and d). This is probably because this set of auxiliary maps explains only $20 \%$ of the original variability.

a

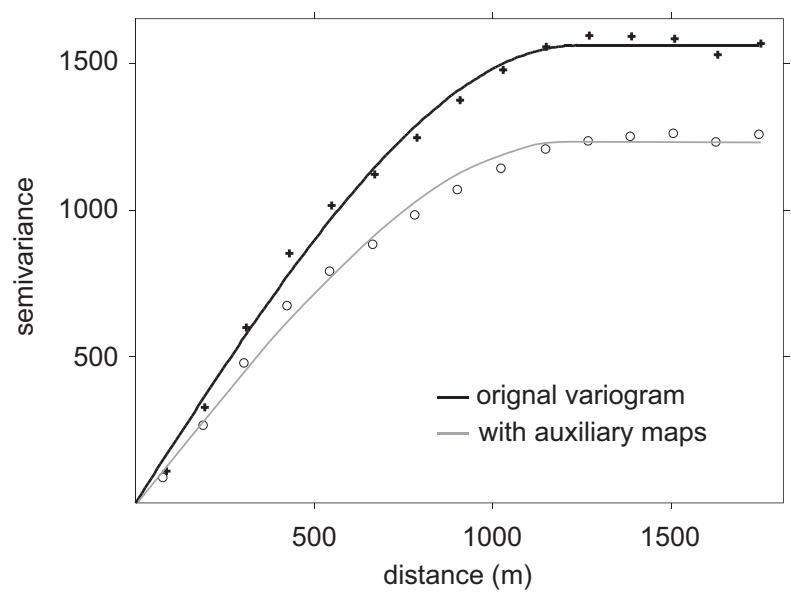

C

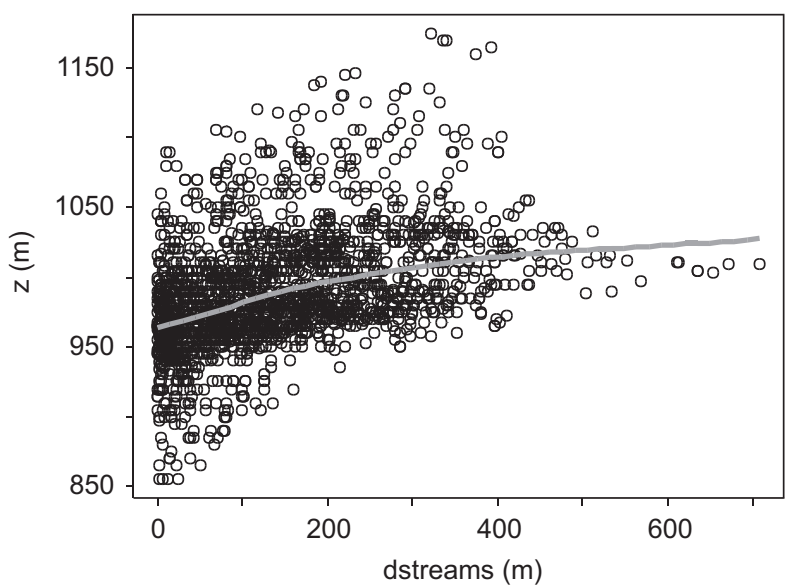

b
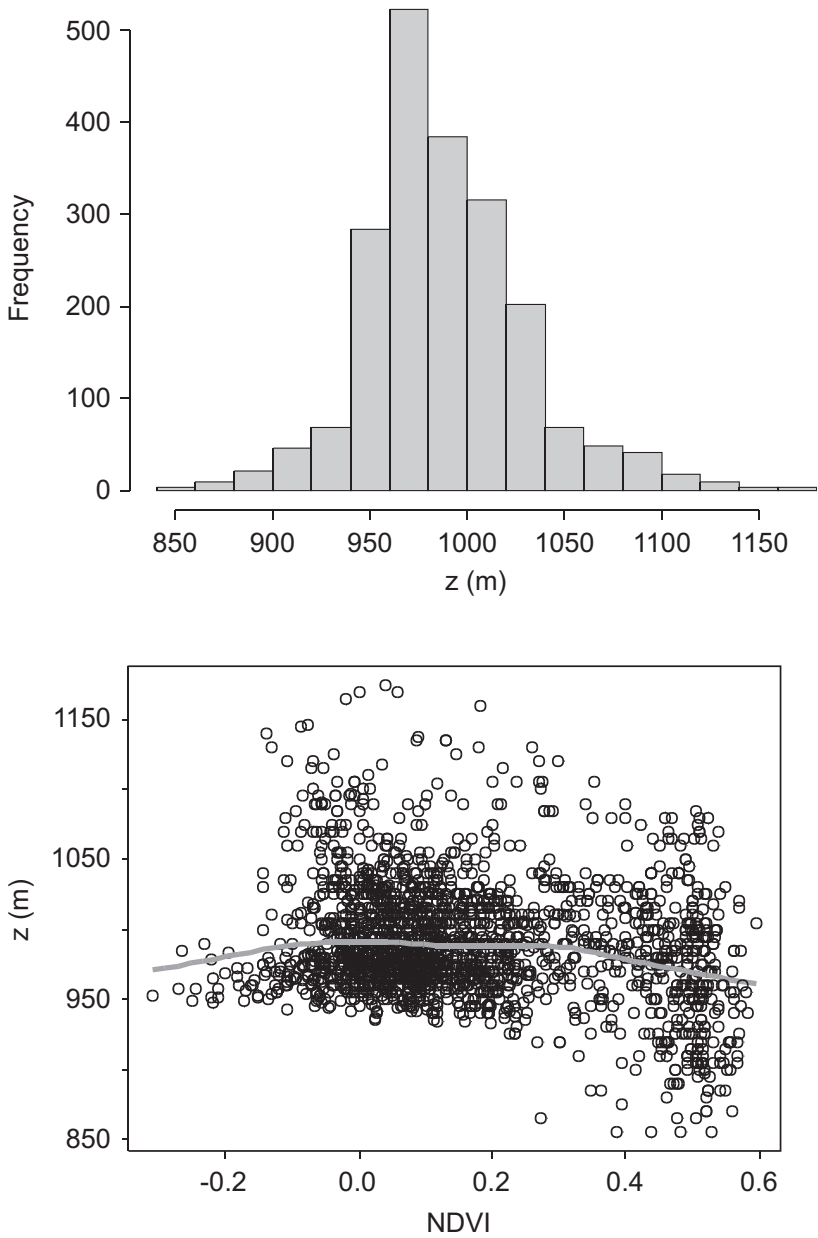

Fig. 3. Elevations used as input for generation of DEMs: (a) fitted variogram models, (b) histogram of elevations and (c) correlation plots: elevation versus dstreams and NDVI. The smooth curve was fitted by loess function and standard smoothness parameter in R. 
a

b

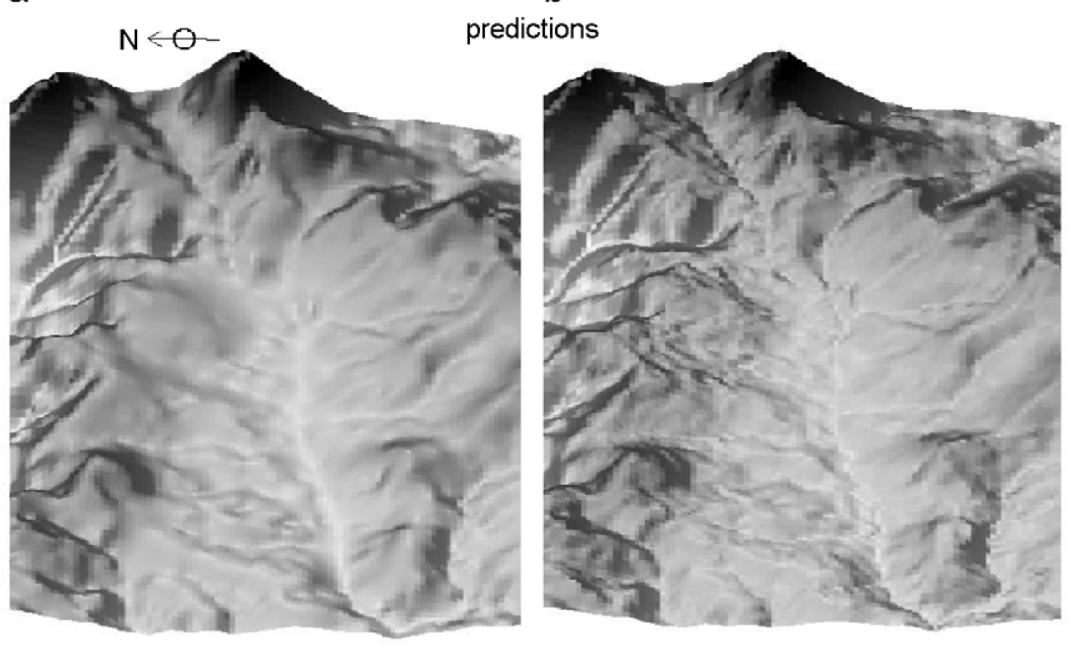

C

d

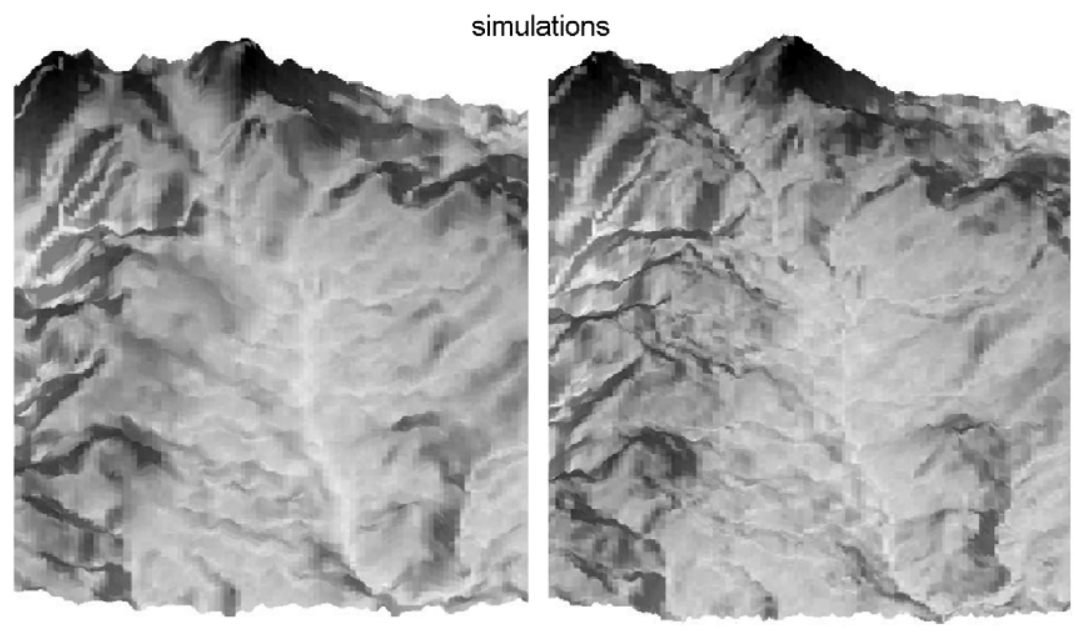

Fig. 4. DEMs generated using krige function in gstat: (a) ordinary kriging predictions (no auxiliary predictors used), (b) regression-kriging predictions, (c) ordinary kriging simulations and (d) regression-kriging simulations. Auxiliary maps used are dstreams and NDVI.

Note also that the simulations, both with and without auxiliary maps are much noisier than the DEMs generated by making predictions. Commonly, people perceive land surface as being smooth, which is often true. However, simulations provide a realization of a land surface which is more realistic - it incorporates also the measurement error and the shortrange variability (Temme et al., 2008). The map shown in Fig. 4d is therefore more suited for further spatial analysis than the map shown in Fig. 4b. The problem is that multiple realizations of a DEMs are needed to make the further spatial analysis realistic, which can often be computationally demanding, hence most of users use only "the most probable (single) realization" of a DEM.

\subsection{DEM error assessment using auxiliary maps}

The deltas assessed at control points for the topo-DEM range from -7.2 to $7.8 \mathrm{~m}$ with an average of $0.18 \mathrm{~m}$ and a standard deviation of $1.2 \mathrm{~m}$. The variogram model of deltas was fitted with a nugget of 1.47 , sill parameter of 0.42 and a range parameter of $332 \mathrm{~m}$ (Fig. 6a). In this case, the short-range variation is significant, i.e. close to the pure nugget effect. The measurement error of the original elevations indicated on the topo-map is about $\pm 1 \mathrm{~m}$, which is in accordance with the vertical accuracy specifications for topographic maps of the scale 1:5000, with contour interval of $5 \mathrm{~m}$ (Committee for Standards and Specifications, 1985). If we interpolate these errors without any auxiliary information, the error surface will show almost no spatial detail (Fig. 6c). 
Regression analysis of errors using auxiliary maps showed that the errors are significantly correlated with terrain complexity (stdev) and terrain orientation (shade). In this case, the predictors explain $27 \%$ of the variability in the 1020 point data set. As expected, the absolute errors are larger on steeper terrains and in shaded areas (Fig. 5). Note that, in the case of the topo-DEM, the two auxiliary maps can only be used to explain local variability of the absolute error values (the heteroscedasticity effect), and not to generate simulations of error surfaces. Note also that in both cases transformations of input variables are needed to obtain relatively linear regression models. The final model used to generate the map in Fig. $6 \mathrm{~d}$ was abs (e) NDVI+log (stdev) +sqrt (shade).

The variogram model of the errors, after including the three predictors, shows smaller nugget (0.36), the sill (0.37) and the range $(114 \mathrm{~m})$ parameters. This proves that the predictors are fairly useful in explaining the spatial distribution of the errors. Consequently, the resulting maps (Fig. 6d) reflect this dependency and the maps of errors are more realistic-much larger errors are now shown in the areas of high relief in the northeastern part of the study area. Compare this map with the geostatistical simulations without auxiliary predictors: if the auxiliary maps are not used, the generated error surface will show much less spatial detail (Fig. 6c).

The assessment of errors in the 3 arcsec SRTM DEM showed that the errors are much larger than for the topo-DEM. The errors range from -38.4 to $59.0 \mathrm{~m}$ with an average of $1.1 \mathrm{~m}$ and a standard deviation of $9.8 \mathrm{~m}$. A histogram of errors (Fig. $7 \mathrm{~b}$ ) shows that the errors are slightly biased toward positive values. The errors can be fitted with an exponential model, zero nugget and sill at 93.6, which corresponds to the global variance. The errors are distinctly correlated with stdev (positively). The regression model explains 26\% of total variation. Note that, this time, no transformation of errors is needed, hence the data sets can be used not only to interpolate absolute errors, but to generate error surface with the help of auxiliary maps (Fig. 7d).
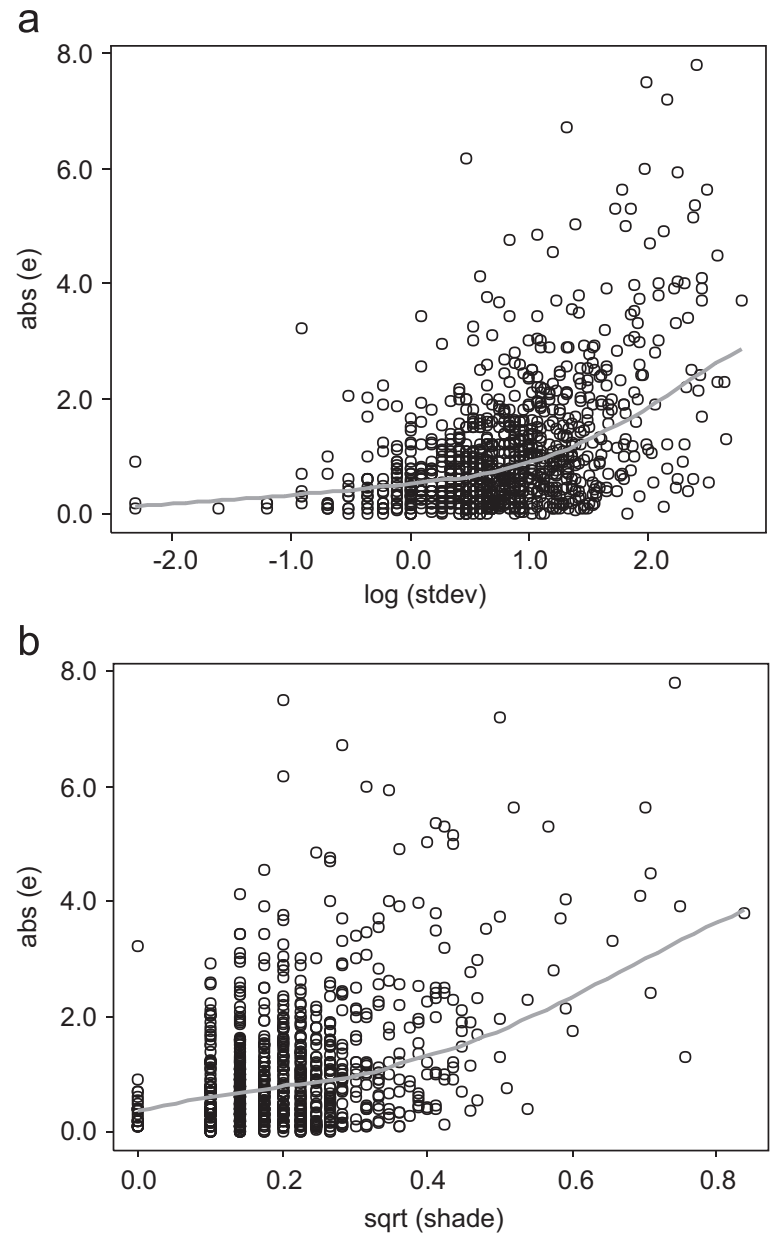

Fig. 5. Correlation plots: absolute errors versus log (stdev) and sqrt (shade) parameters. A smooth curve was fitted by loess function and standard smoothness parameter in $\mathrm{R}$. 
a

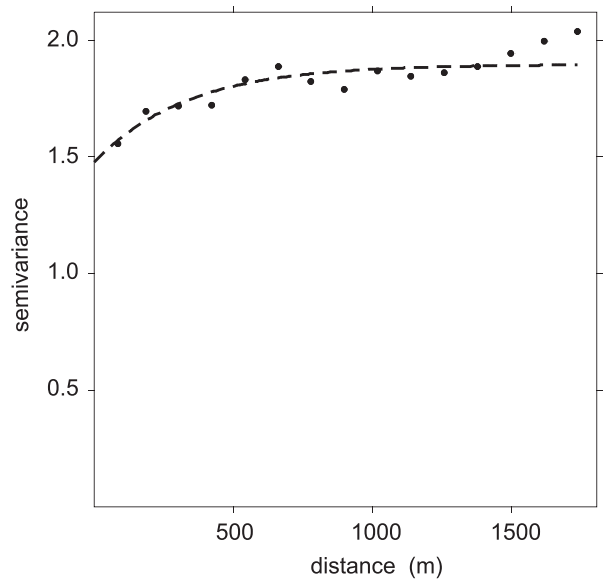

C

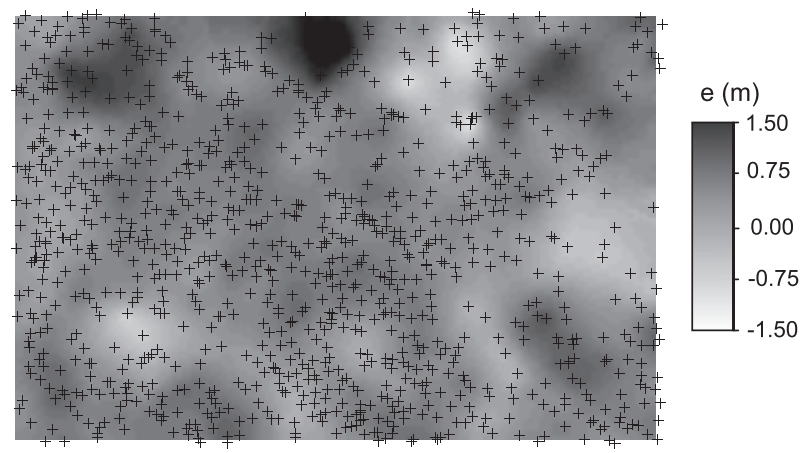

b

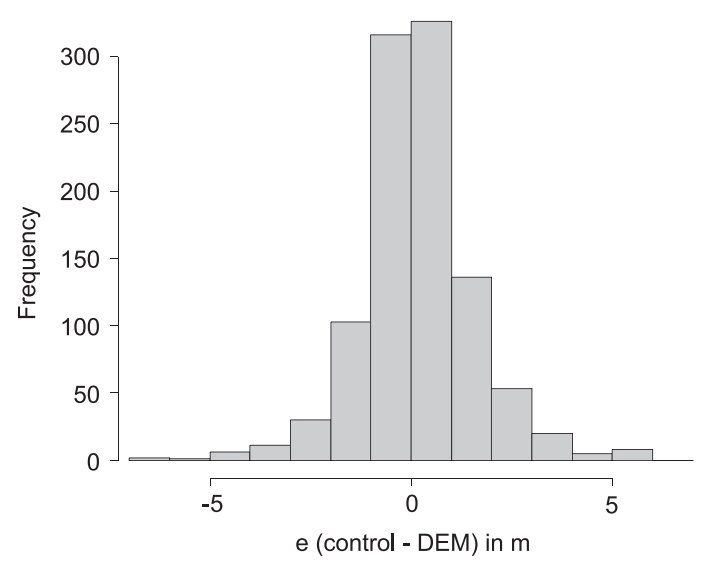

d

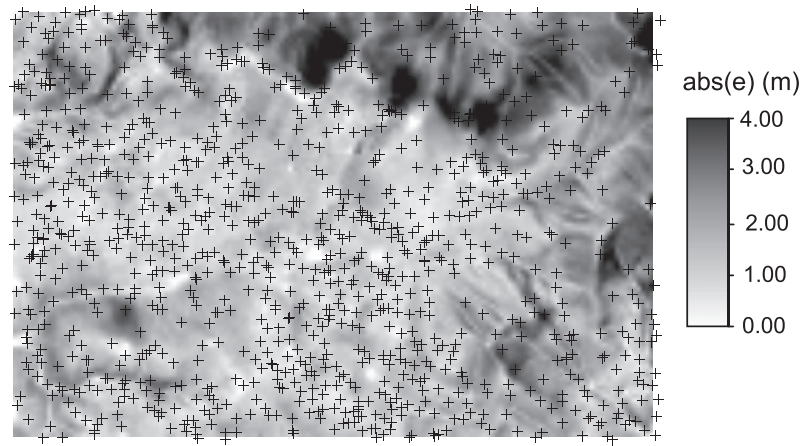

Fig. 6. Error assessment for topo-DEM using control data set: (a) fitted variogram model, (b) histograms of errors (e), (c) interpolated error surface using pure ordinary kriging and (d) using regression-kriging and auxiliary information.

\subsection{Downscaling of the SRTM DEM using auxiliary maps}

In the last exercise, we used auxiliary maps to downscale an existing coarse SRTM DEM ( 3 arcsec or $\sim 90 \mathrm{~m}$ ) to a finer scale of $30 \mathrm{~m}$. The principle was the same as when working with the elevation data set used to directly generate DEMs-the grid nodes (1700 in total) are used as points and dstreams and NDVI maps are used as auxiliary maps. However, in this example the grid nodes do not represent punctual measurements but block measurements (support size equals grid spacing between grid nodes). Consequently, the fitted variogram was a Bessel model with no nugget variation $\left(C_{0}=0\right)$, sill variation of 1251 and effective range at around $1450 \mathrm{~m}(R=474 \mathrm{~m})$. Also, the regression model between SRTM elevations and auxiliary maps is much less distinct (adj. $R^{2}=0.10$; compared to 0.20 ). The variogram model of the SRTM DEM is not suitable for estimation of values at finer support size, hence we used an "empirical" variogram model, i.e. the model fitted in Fig. 3a.

The results of downscaling are shown in Fig. 8b; compared with the original SRTM DEM shown in Fig. 8a. The downscaled DEM is still much smoother than it should be (Fig. $4 \mathrm{~b}$ ), however the streams are now better represented and the RMSE at control locations is lower (10.5 vs. $11.2 \mathrm{~m}$ ). Downscaling is obviously possible only to a certain degree with this limited set of auxiliary maps. Note also that, if the field-measured point heights (Fig. 3a) did show nugget variation, this information would have been permanently lost in the SRTM DEM. In this case study, we can assume that the original variable varies smooth (Fig. 3a), so that downscaling is definitively possible as long as we can obtain more-detailed auxiliary information (streams lines, topographic breaks, etc.) than the original SRTM DEM shows.

\section{Discussion and conclusions}

The use of kriging in geomorphometry to generate DEMs has been criticized by many (Wood and Fisher, 1993; Mitas and Mitasova, 1999; Li et al., 2005), mainly because it leads to many artifacts, it oversmooths elevations and it is very sensitive 
a

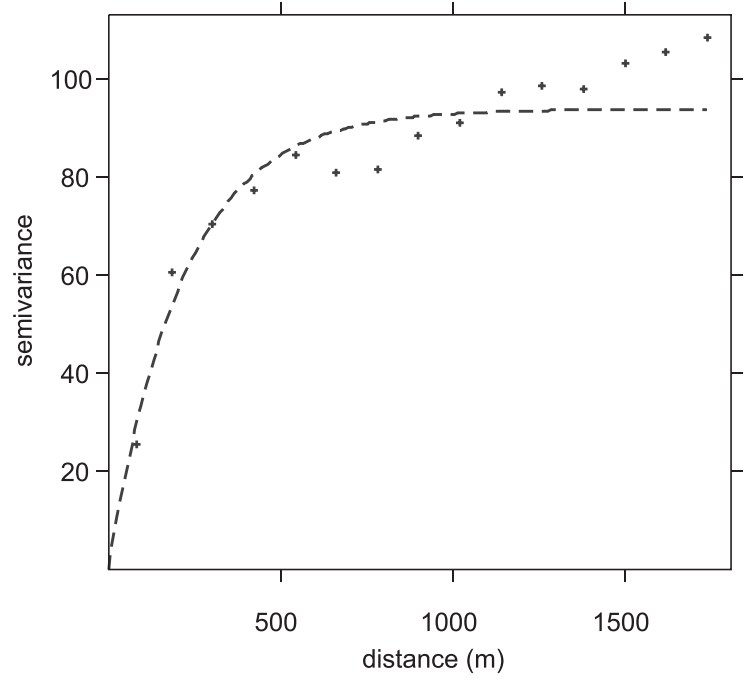

C

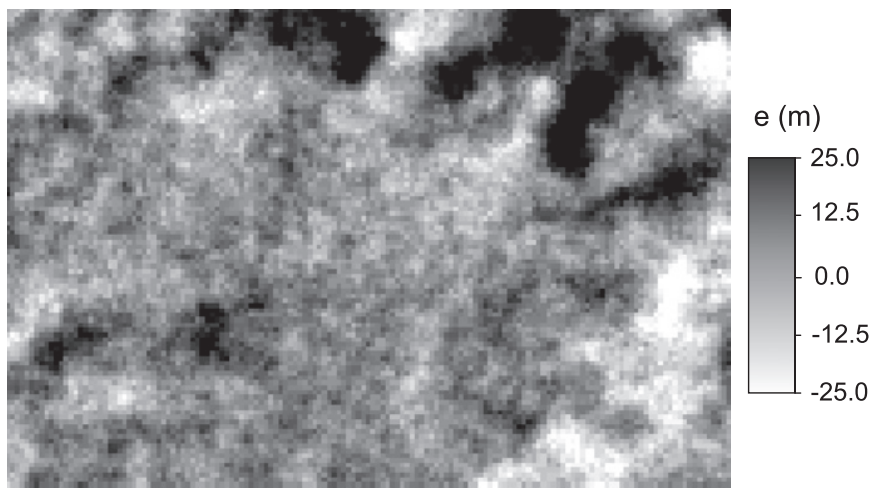

b

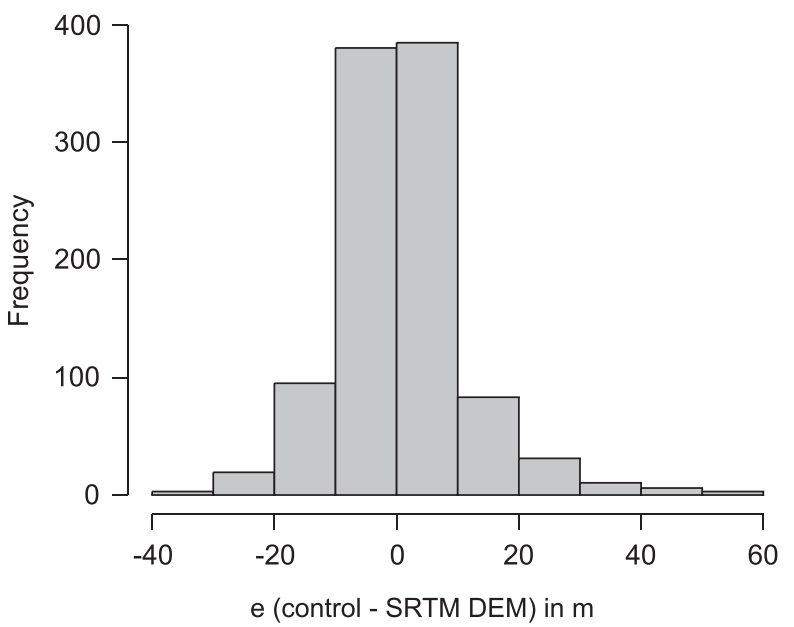

d

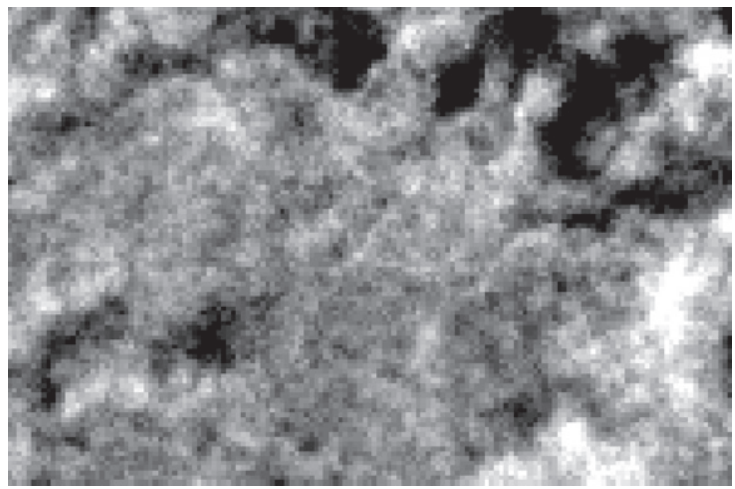

Fig. 7. Error assessment for SRTM DEM using control data set: (a) fitted variogram model, (b) histograms of errors (e), (c) simulated error surface using pure ordinary kriging and (d) using regression-kriging and auxiliary information.

to sampling density and local extreme values. So far, splines have been used in geomorphometry as a preferred technique to generate DEMs or to filter local errors (Mitasova et al., 2005). This case study has shown that realistic DEMs (especially considering the hydrological features) can also be generated using regression-kriging and auxiliary maps. In addition, we have demonstrated that auxiliary maps can help explain spatial distribution of errors in DEMs. In this case study, errors were positively correlated with terrain complexity (stdev), hillshading (shade) and with vegetation (NDVI). This confirms the previous results of Fisher (1998), Holmes et al. (2000, p. 162) and Carlisle (2005). Finally, we showed that regressionkriging and auxiliary maps can be used to downscale existing coarse DEMs. This will work well especially if fine-scale vector maps of drainage networks and topographic breaks and a variogram model of elevation measurements at finer support size are available.

The methodology proposed in this paper is fit for use with any type of terrain, provided that both auxiliary maps and point data sets are available and suited for the area of interest. Most common requirements are that the auxiliary layers should have a complementary effective scale, and that the samples are representative for the area of interest. The remaining issues of using regression-kriging in geomorphometry are the computation efficiency and proper selection of auxiliary maps. In this exercise, for the reasons of simplicity, we have used a rather small number of auxiliary maps and a relatively small data set (15,000 grid nodes). Even this small exercise will take several minutes to perform all computations. We assume that the list of maps can be extended several times and methodology applied in real-life projects, but the computational complexity would then become a real cumbersome. For example, in the case of LiDAR data, the density of point measurements is very high and one will work with huge point data sets that are often very difficult to run geostatistical analysis because large matrices need to be inverted.

Therefore, we anticipate that the major challenges for further refinement of these techniques will be a proper design and use of auxiliary data for such analysis and implementation of local regression-kriging methods, i.e. fitting of variograms 


\section{a}

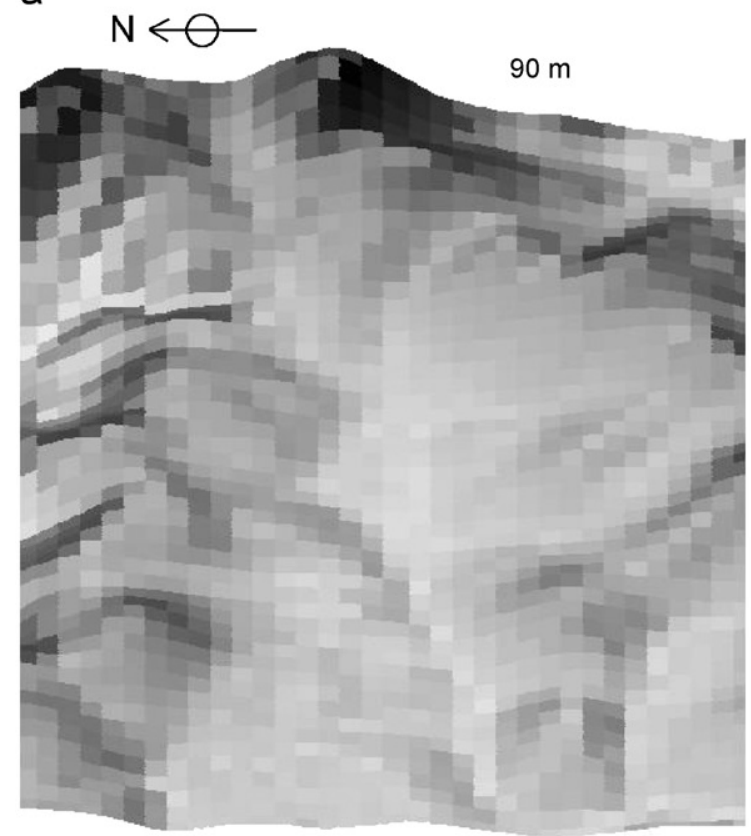

$\mathrm{b}$

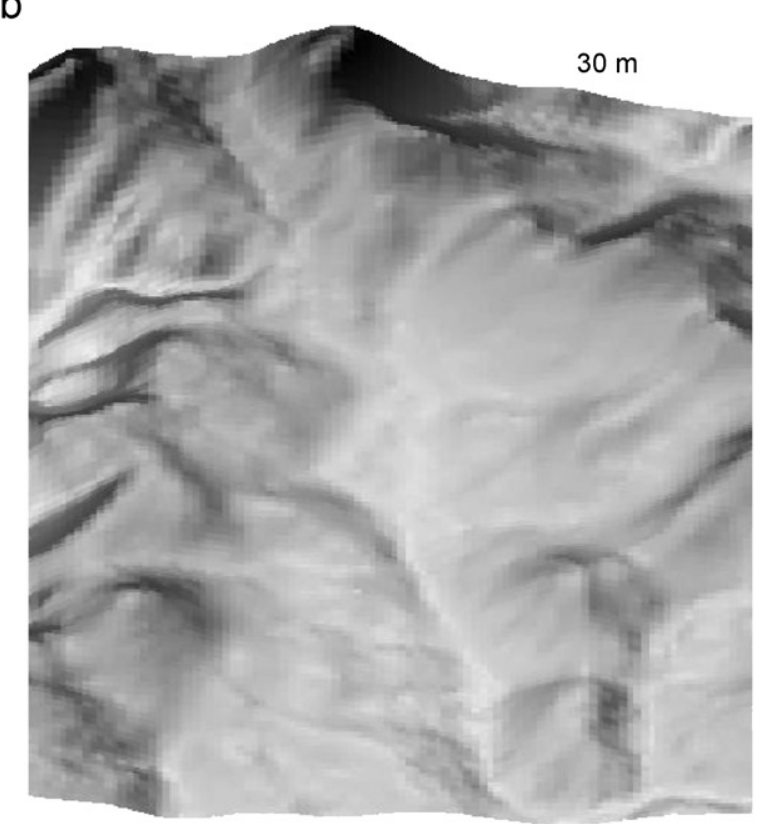

Fig. 8. Result of downscaling $90 \mathrm{~m}$ SRTM DEM (a) to a $30 \mathrm{~m}$ grid (b) using auxiliary maps dstreams and NDVI. Compare with Fig. 4.

and regression models in a moving window. Considering the extension of potential auxiliary maps of interest for geostatistical modeling of topography, three major groups will play an increasing role:

(1) Hydrological maps:

- stream line data;

- water stagnation areas (soil-water content images);

- seashore/lakes line;

(2) Land cover maps:

- canopy height;

- leaf area index;

- land cover classes; 
(3) Geomorphological maps:

- surface roughness maps;

- physiographic breaks;

- ridges and terraces.

Certainly, this list can be extended and refined. A lot of topography-connected information can be derived from remotesensing multi and hyper spectral images. We have used only one index (NDVI), but we assume that more useful indices/ objects can be designed to represent changes in topography such as shading-based indices, drainage patterns, ridge-lines, topographic breaks. All these can be derived using automated (pattern recognition) techniques, which can significantly speed up processing for large areas.

Note also that many auxiliary maps will mutually overlap in information and value. For example, in this study, we have used some auxiliary maps that are derived from the same DEM (e.g. stdev, shade) that is then being analyzed for errors. Ideally, auxiliary maps should be only GIS layers produced independently from the sampled elevations-e.g. remotesensing images, topographic features, thematic maps, etc. Where this is not possible, auxiliary maps can be derived from an existing DEM, provided that this DEM is generated using independent elevation measurements. Care needs to be taken not to employ auxiliary maps which are only indirectly or accidentally connected with the variations in topography. Otherwise unrealistic simulations can be generated, of even poorer quality than if only standard DEM generation techniques are used.

We can conclude that the hybrid geostatistical techniques such as regression-kriging offer potentially significant advantages over standard DEM generation techniques such as splines. Especially because regression-kriging offers an objective estimation of the model parameters and, hence, allows data automation. Such tools will be more and more attractive for handling rich LiDAR and radar-based topographic data, both to analyze their inherent geostatistical properties and to generate DEMs fit-for-use in various environmental and earth science applications. Now it is time to refine these tools and make them operational for real-life projects.

\section{References}

Box, G.E.P., Muller, M.E., 1958. A note on the generation of random normal deviates. The Annals of Mathematical Statistics 29 (2), 610-611.

Carlisle, B.H., 2005. Modelling the spatial distribution of DEM error. Transactions in GIS 9, 521-540.

Christensen, R., 2001. Linear Models for Multivariate, Time Series, and Spatial Data, second ed. Springer Text in Statistics. Springer, New York, 393pp.

Committee for Standards and Specifications, 1985. Accuracy specifications for large-scale line maps. Photogrammetric Engineering and Remote Sensing 51 (2), 195-199.

Endreny, T.A., Wood, E.F., 2001. Representing elevation uncertainty in runoff modelling and flowpath mapping. Hydrological Processes 15, $2223-2236$.

Evans, I.S., 1972. General geomorphometry, derivatives of altitude, and descriptive statistics. In: Chorley, R.J. (Ed.), Spatial Analysis in Geomorphology. Harper \& Row, New York, pp. 17-90.

Evans, J.S., Hudak, A.T., 2007. A multiscale curvature filter for identifying ground returns from discrete return lidar in forested environments. IEEE Transactions on Geoscience and Remote Sensing 45 (4), 1029-1038.

Fisher, P., 1992. First experiments in viewshed uncertainty: simulating fuzzy viewsheds. Photogrammetric Engineering and Remote Sensing 58 (3), 345-352.

Fisher, P., 1998. Improved modeling of elevation error with geostatistics. GeoInformatica 2 (3), 215-233.

Goovaerts, P., 1997. Geostatistics for Natural Resources Evaluation (Applied Geostatistics). Oxford University Press, New York, 496pp.

Grohmann, C.H., 2004. Morphometric analysis in geographic information systems: applications of free software GRASS and R. Computers \& Geosciences 30, 1055-1067.

Hengl, T., 2007. A practical guide to geostatistical mapping of environmental variables. EUR 22904 EN. Office for Official Publications of the European Communities, Luxembourg, 143pp.

Hengl, T., Reuter, H.I. (Eds.), 2008. Geomorphometry: concepts, software, applications. In: Developments in Soil Science, vol. 33. Elsevier, Amsterdam, in press.

Heuvelink, G.B.M., 1998. Error Propagation in Environmental Modelling with GIS. Taylor \& Francis, London, UK, 144pp.

Holmes, K.W., Chadwick, O.A., Kyriakidis, P.C., 2000. Error in a USGS 30m digital elevation model and its impact on digital terrain modeling. Journal of Hydrology 233, 154-173.

Hunter, G.J., Goodchild, M.F., 1997. Modeling the uncertainty of slope and aspect estimates derived from spatial databases. Geographical Analysis 29 (1), 35-49.

Hutchinson, M.F., 1989. A new procedure for gridding elevation and stream line data with automatic removal of spurious pits. Journal of Hydrology 106 $211-232$.

Hutchinson, M.F., 1996. A locally adaptive approach to the interpolation of digital elevation models. In: Proceedings of the Third International Conference Workshop on Integrating GIS and Environmental Modeling. National Center for Geographic Information and Analysis, Santa Barbara, CA, 6pp.

Kyriakidis, P.C., Shortridge, A.M., Goodchild, M.F., 1999. Geostatistics for conflation and accuracy assessment of digital elevation models. International Journal of Geographical Information Science 13 (7), 677-708.

Li, Z., Zhu, Q., Gold, C., 2005. Digital Terrain Modeling: Principles and Methodology. CRC Press, Boca Raton, FL, 319pp.

Lloyd, C.D., Atkinson, P.M., 1998. Scale and the spatial structure of landform: optimizing sampling strategies with geostatistics. In: Proceedings of the Third International Conference on GeoComputation, University of Bristol, UK, 17-19 September 1998. University of Bristol, Bristol, UK, 16pp.

Lloyd, C.D., Atkinson, P.M., 2002. Deriving DSMs from LiDAR data with kriging. International Journal of Remote Sensing 23 (12), $2519-2524$

Mitas, L., Mitasova, H., 1999. Spatial interpolation. In: Longley, P., Goodchild, M.F., Maguire, D.J., Rhind, D.W. (Eds.), Geographical Information Systems: Principles Techniques Management and Applications, vol. 1. Wiley, New York, pp. 481-492.

Mitasova, H., Mitas, L., Harmon, R.S., 2005. Simultaneous spline approximation and topographic analysis for Lidar elevation data in open-source GIS. IEEE Geoscience and Remote Sensing Letters 2, 375-379.

Oksanen, J., 2006a. Digital elevation model error in terrain analysis. Ph.D. Dissertation, Faculty of Science, University of Helsinki, Finland, 59pp.

Oksanen, J.J.A., 2006b. Uncovering the statistical and spatial characteristics of fine toposcale DEM error. International Journal of Geographical Information Science 20 (4), 345-356.

Oksanen, J., Sarjakoski, T., 2005. Error propagation of DEM-based surface derivatives. Computers \& Geosciences 31 (8), $1015-1027$.

Pebesma, E.J., 2004. Multivariable geostatistics in S: the gstat package. Computers \& Geosciences 30 (7), $683-691$.

Pebesma, E.J., Bivand, R.S., 2005. Classes and methods for spatial data in R. R News 5 (2), 9-13. 
Pike, R.J., 1995. Geomorphometry_progress, practice, and prospect. Zeitschrift für Geomorphologie Supplementband 101, $221-238$.

Raaflaub, L.D., Collins, M.J., 2006. The effect of error in gridded digital elevation models on the estimation of topographic parameters. Environmental Modelling \& Software 21, 710-732.

Selige, T., Böhner, J., Ringeler, A., 2006. Processing of SRTM X-SAR data to correct interferometric elevation models for land surface applications. In: Böhner, J., McCloy, K.R., Strobl, J. (Eds.), SAGA-Analyses and Modelling Applications, vol. 115. Verlag Erich Goltze GmbH, pp. 97-104.

Temme, A.J.A.M., Heuvelink, G.B.M., Schoorl, J.M., Claessens, L., 2008. Geostatistical simulation and error propagation in geomorphometry. In: Hengl, T., Reuter, H.I. (Eds.), Geomorphometry: Concepts, Software, Applications, volume 33: Developments in Soil Science. Elsevier, Amsterdam, in press. Wilson, J.P., Gallant, J.C. (Eds.), 2000. Terrain Analysis: Principles and Applications. Wiley, New York, 303pp.

Wood, J., Fisher, P.F., 1993. Assessing interpolation accuracy in elevation models. IEEE Computer Graphics and Applications 13 (2), $48-56$. 